\title{
On-Line Paging against Adversarially Biased Random Inputs
}

\author{
Neal E. Young*
}

\begin{abstract}
In evaluating an algorithm, worst-case analysis can be overly pessimistic. Average-case analysis can be overly optimistic. An intermediate approach is to show that an algorithm does well on a broad class of input distributions. Koutsoupias and Papadimitriou [10] recently analyzed the least-recently-used (LRU) paging strategy in this manner, analyzing its performance on an input sequence generated by a so-called diffuse adversary — one that must choose each request probabilitistically so that no page is chosen with probability more than some fixed $\epsilon>0$. They showed that LRU achieves the optimal competitive ratio (for deterministic on-line algorithms), but they didn't determine the actual ratio.

In this paper we estimate the optimal ratios within roughly a factor of two for both deterministic strategies (e.g. least-recently-used and firstin-first-out) and randomized strategies. Around the threshold $\epsilon \approx 1 / k$ (where $k$ is the cache size), the optimal ratios are both $\Theta(\ln k)$. Below the threshold the ratios tend rapidly to $O(1)$. Above the threshold the ratio is unchanged for randomized strategies but tends rapidly to $\Theta(k)$ for deterministic ones.

We also give an alternate proof of the optimality of LRU.
\end{abstract}

\section{Introduction and Background}

The paging problem was originally studied in the context of two-level virtual memory systems composed of a large, slow-access memory augmented with a cache (a small, fast-access memory, holding likely-to-be accessed pages in order to minimize access time).

This paper concerns the following standard abstraction of this simple and common problem. The input is an integer $k$ and a finite sequence $s=s_{1} s_{2} \ldots s_{n}$ of requests. The parameter $k$ is called the cache size. The output is a schedule - a sequence $S_{1} S_{2} \ldots S_{n}$ of sets, where each set is of size at most $k$, and each $S_{t}$ contains $s_{t}$. Each request $s_{t}$ is said to occur at time $t$. The items in $S_{t}$ are said to be in the cache after time $t$ up to and including time $t+1$. An item is said to be evicted at time $t$ if the item is in $S_{t-1}$ but not in $S_{t}$. The cost of the

*Dartmouth College, Hanover NH 03755, ney@cs.dartmouth.edu. Research partially funded by NSF CAREER award CCR-9720664. 
schedule is number of evictions. A schedule for an input is optimal if it achieves the minimum possible cost.

Next we define the paging algorithms considered in this paper. Each evicts pages only when the cache is full and does not contain the requested item. Least-recently-used (LRU) evicts the item whose most recent request is the least recent among all items in the cache. First-in-first-out (FIFO) evicts the item that has been in the cache the longest. Flush-when-full (FWF) evicts all items in the cache. The randomized marking algorithm (RMARK [5]) operates as follows. After an item is requested, it is marked. When an item must be evicted, a nonmarked item is chosen uniformly at random, with the caveat that if all items in the cache are marked, then all marks are first erased. By a deterministic marking algorithm, we mean any deterministic algorithm that maintains marks as RMARK does, and evicts only unmarked items. LRU, FIFO, and FWF are examples. By a lazy deterministic marking algorithm (DMARK), we mean a deterministic marking algorithm that evicts an item only when necessary, and then only one item. This additional requirement excludes FWF.

An algorithm for the problem is on-line if, for any request sequence and any request in that sequence, the items in the cache after the request are independent of later requests. In many contexts, on-line algorithms are necessary, but online algorithms are necessarily sub-optimal on some request sequences. Hence, a natural question is how on-line algorithms can be effectively analyzed and compared.

This paper is concerned with a generalization of the standard competitive analysis [14] of on-line algorithms. The standard model measures the quality of an algorithm $A$ by its competitive ratio: the minimum (to be precise, infimum) $c$ such that, for some constant $b$, for all request sequences $s$,

$$
A(s) \leq c \cdot \operatorname{OpT}(s)+b .
$$

Here $A(s)$ denotes the cost of the schedule produced by $A$ on input $s ; \operatorname{Opt}(s)$ denotes the cost of an optimal schedule. If $A$ is a randomized algorithm, then $A(s)$ denotes the expected cost of $A$ on input $s$. Note that $k$ is an implicit, and fixed, parameter in these definitions. Standard competitive analysis is a worst-case type of analysis, in contrast to much of the earlier work on paging, which is concerned with average-case analysis?

In the standard competitive-analysis framework the following results are known. Any deterministic marking algorithm, including LRU, FIFO, and FwF, has a competitive ratio of $k$; the ratio $k$ is the best possible for any deterministic on-line strategy 14, 2]. The randomized marking algorithm RMARK has a competitive ratio of $2 H(k)-1$ [5, 1, where $H(k) \doteq \sum_{1}^{k} 1 / i \approx \ln (1+k)$. PARTITION [12] and Equitable [1], more complicated randomized algorithms, each have competitive ratio $H(k)$. No randomized strategy can have a better ratio than $H(k)$ 河.

\footnotetext{
1 At least one work preceding competitive analysis blends average-case and worstcase analysis. It considers input sequences where each request is chosen from a fixed but unknown distribution on the pages, and compares known paging strategies to the optimal on-line strategy for that distribution.
} 
Largely due to the unrealistic magnitude of the optimal competitive ratios [16], many variations on the standard model have been considered (e.g. [3, 4, 8, 6, 9, 11, 16, 17]). For a survey on competitive analysis of paging, we refer the reader to the recent book by Borodin and El-Yaniv [2, ch's 3-5].

This paper concerns the following generalization of the standard model, recently proposed by Koutsoupias and Papadimitriou [10. For any class $\Delta$ of distributions on the input sequences and any deterministic or randomized algorithm $A$, define $\mathcal{R}(\Delta, A)$, the competitive ratio of $A$ against the $\Delta$-diffuse adversary, to be the minimum (again, to be precise, infimum) $c$ such that for each distribution $D$ in $\Delta$, there is a constant $b$ such that

$$
\mathrm{E}_{D}[A(r)] \leq c \cdot \mathrm{E}_{D}[\mathrm{OPT}(r)]+b .
$$

Here $r$ is a random sequence chosen according to $D$. Define the optimal ratio for deterministic on-line algorithms (against the $\Delta$-diffuse adversary) to be

$$
\mathcal{R}(\Delta) \doteq \inf _{A} \mathcal{R}(\Delta, A),
$$

where $A$ ranges over all deterministic on-line algorithms. Analogously, define the optimal ratio for randomized on-line algorithms (against the $\Delta$-diffuse adversary) to be

$$
\mathcal{R} \mathcal{R}(\Delta) \doteq \inf _{A_{R}} \mathcal{R}\left(\Delta, A_{R}\right)
$$

where $A_{R}$ ranges over all randomized on-line algorithms.

The particular class of distributions considered by Koutsoupias and Papadimitriou is denoted $\Delta_{\epsilon}$ and is defined as follows. Any distribution $D$ specifies, for each item $x$ and sequence of requests $s$, the probability $\operatorname{Pr}_{D}(x \mid s)$ that the next request of the random sequence $r$ is $x$ given that the sequence so far is $s$. Then $\Delta_{\epsilon}$ contains those distributions $D$ such that, for any request sequence $s$ and item $x, \operatorname{Pr}_{D}(x \mid s) \leq \epsilon$. The parameter $\epsilon$ is a measure of the inherent uncertainty of each request. Koutsoupias and Papadimitriou show that LRU achieves the optimal ratio in this model (i.e. $\mathcal{R}\left(\Delta_{\epsilon}, \mathrm{LRU}\right)=\mathcal{R}\left(\Delta_{\epsilon}\right)$ ), but they leave open the question of what the ratio is.

Here we estimate the optimal ratios within roughly a factor of two, for both deterministic and randomized algorithms. Here is our main theorem.

Theorem 1 Define

$$
\Phi(\epsilon, k) \doteq 1+\sum_{i=1}^{k-1} \max \left\{\epsilon^{-1}-i, 1\right\}^{-1} .
$$

For any $\epsilon$, let $\epsilon^{\prime}=1 /\left\lceil\epsilon^{-1}\right\rceil$. The competitive ratios of deterministic $(\mathcal{R})$ and randomized $(\mathcal{R} \mathcal{R})$ on-line algorithms against the $\Delta_{\epsilon}$-diffuse adversary are bounded as follows: 


\begin{tabular}{|c|c|c|c|c|}
\hline \multirow[b]{2}{*}{ range } & \multicolumn{2}{|c|}{ deterministic $-\mathcal{R}\left(\Delta_{\epsilon}\right)$} & \multicolumn{2}{|c|}{ randomized $-\mathcal{R} \mathcal{R}\left(\Delta_{\epsilon}\right)$} \\
\hline & lower bound & $\overline{\text { upper bound }}$ & lower bound & 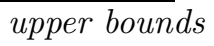 \\
\hline$\epsilon \leq 1 /(k+1)$ & $\Phi(\epsilon, k)-1$ & $2 \Phi(\epsilon, k)$ & $\Phi\left(\epsilon^{\prime}, k\right)-1$ & $2 \Phi(\epsilon, k)$ \\
\hline$\epsilon \geq 1 /(k+1)$ & $\Phi(\epsilon, k)$ & $2 \Phi(\epsilon, k)$ & $H(k)$ & $H(k)$ \\
\hline
\end{tabular}

The upper bound $2 \Phi$ for deterministic algorithms holds for any lazy marking algorithm (e.g. LRU, FIFO) but not for FWF. The upper bound $H(k)$ for randomized algorithms holds for PARTITION and EQUITABLE. The weaker upper bound $2 H(k)-1$ holds for RMARK.

In all cases except one, the competitive ratios of (lazy) deterministic and randomized marking algorithms are at least $\Phi-1$ and at most $2 \Phi$. The exception is that for $\epsilon$ above the threshold $1 /(k+1)$, the randomized ratio is $H(k)$ (independently of $\epsilon$ ). To understand the behavior of the function $\Phi$, consider the case $\epsilon=1 / n$ for some integer $n$. Then

$$
\Phi(1 / n, k)=1+H(n-1)+\left\{\begin{array}{cc}
-H(n-k) & \text { when } n \geq k, \\
k-n & \text { when } n \leq k .
\end{array}\right.
$$

Recall that $H(k) \doteq \sum_{1}^{k} 1 / i \approx \ln (k+1)$. The threshold of $\Phi$ around $\epsilon \approx 1 / k$ is very sharp:

$$
\Phi(\epsilon, k) \text { is } \begin{cases}\leq 1+\ln \frac{1}{\delta} & \text { when } \epsilon=(1-\delta) / k \\ \approx \ln k & \text { when } \epsilon=1 / k \\ \geq k \frac{\delta}{1+\delta} & \text { when } \epsilon=(1+\delta) / k\end{cases}
$$

\section{Technical Overview}

We refine an existing worst-case competitive analysis for paging [14, 5, 2] to take into account the probabilistic restrictions on the adversary. We call this particular analysis the factor-two-analysis because for our purposes (and when used to analyze the randomized marking algorithm [5]) it (at best) can approximate OpT only within a factor of two.

\subsection{Review of Factor-Two-Analysis in the Standard Model}

Let $A$ be any paging algorithm and let $s=s_{1} s_{2} \ldots s_{n}$ be any sequence of requests. The phases of $s$ partition the times $\{1,2, \ldots, n\}$ into intervals as follows. Define $t(1)=1$. For $\ell \in \mathbb{N}$ inductively define

$$
t(\ell+1) \doteq 1+\max \left\{j \leq n:\left|\left\{s_{t(\ell)}, s_{t(\ell)+1}, s_{t(\ell)+2}, \ldots, s_{j}\right\}\right| \leq k\right\} .
$$

For each $\ell \in \mathbb{N}$ such that $t(\ell) \leq n$, the $\ell$ th phase of $s$ is defined to be the time interval $\{t(\ell), t(\ell)+1, \ldots, t(\ell+1)-1\}$. Thus, during each phase except the last, $k$ distinct items are requested. 
In the context of a particular time $t$, the current request refers to the request $s_{t}$. This phase or, synonymously, the current phase means the phase containing the time $t$. An item is requested previously in this phase if it is requested during this phase before time $t$. In the additional context of a particular schedule $S=S_{1} S_{2} \ldots S_{n}$ for $s$, the cache refers to the set $S_{t-1}$ of items in the cache before request $t$. Then at each time $t$, each item is classified with respect to its status before request $s_{t}$ as follows:

new - not requested previously in this phase or in the last phase.

old - requested during the last phase, but not previously in this phase.

redundant - requested previously in this phase.

worrisome - requested in the last phase or previously in this phase, but not in the on-line algorithm's cache.

Each request is classified as well, according to the status of the requested item. For instance, a request $s_{t}$ is new if the requested item was new after request $s_{t-1}$. Each phase (except possibly the last) has $k$ non-redundant requests, each one of which is either new or old. Define

new $(s)$ - the total number of new requests in sequence $s$.

new_in_ph $(\ell)$ - (in the context of some sequence) the total number of new requests in the $\ell$ th phase of the sequence. Here $\ell$ is any positive integer. If $\ell=0$ or there is no $\ell$ th phase, define new_in_ph $(\ell)$ to be 0 .

The relevance of the new requests is as follows.

Lemma $1([5$, 15] $)$ new $(s) / 2 \leq \operatorname{Opt}(s) \leq$ new $(s)$

Proof: Consider the $(\ell-1)$ st and $\ell$ th phases of $s$ for any $\ell$. The number of distinct items requested in the two phases is $k+$ new_in_ph $(\ell)$. Thus, the number of evictions incurred by OPT during the two phases is at least new_in_ph $(\ell)$ and

$$
\begin{aligned}
\operatorname{Opt}(s) & \geq \max \left\{\sum_{\ell \text { odd }} \text { new_in_ph }(\ell), \sum_{\ell \text { even }} \text { new_in_ph }(\ell)\right\} \\
& \geq \sum_{\ell} \text { new_in_ph }(\ell) / 2=\operatorname{new}(s) / 2 .
\end{aligned}
$$

On the other hand, the following schedule costs at most new $(s)$. At the beginning of each phase, evict those items that are not requested during the phase and bring in the items that are not in the cache but are requested during the phase. After each phase ends, the items requested during that phase are in the cache, so the number of evictions in the next phase is just the number of new requests in that phase. Thus, the cost of this schedule is new $(s)$. Since the schedule produced by Opт is at least as $\operatorname{good}, \operatorname{Opt}(s) \leq \operatorname{new}(s)$. 
By the amortized cost incurred by OpT during a phase, we mean half the number of new requests in that phase. By the lemma above, the total cost incurred by OpT is at least the total of these amortized costs and at most twice the total. To show bounds on the competitive ratio of $A$, we use the standard method of bounding the cost incurred by $A$ during a phase divided by the amortized cost incurred by OPT during the phase. For instance, if this ratio is at most $c$ for each phase of a sequence $s$, then it follows immediately that $A(s) \leq c \cdot \operatorname{Opt}(s)$.

One intuition for understanding RMARK and other marking algorithms such as LRU, FIFO, and even FWF is that they are emulating the schedule described in the proof above that $\operatorname{Opt}(s) \leq$ new $(s)$. That is, during each phase, the "goal" (intuitively speaking) is to get the items that will be requested during the phase into the cache. From this point of view, once an item is requested during a phase, it should be kept in the cache. This is the principle that defines a deterministic marking algorithm.

If this principle is followed, then only non-redundant requests can cause evictions. Since the phase ends after $k$ non-redundant requests, any deterministic marking algorithm incurs a cost of at most $k$ during the phase. This means that in the standard model, the competitive ratio is at most $k$ (OpT also incurs at least one eviction per phase). Conversely, the adversary can force a ratio of $k$ against a deterministic on-line algorithm by making one new request each phase and then making $k-1$ requests, each to whichever old item is not currently in the cache.

\subsection{Factor-Two-Analysis for the Diffuse Adversary}

In the standard model, the adversary can force each old request (i.e., each non-redundant request to an item requested in the previous phase) to cause an eviction. In the diffuse adversary model, this is not so, because the adversary can only assign $\epsilon$ probability to each item. The adversary may have to assign probability to redundant and/or new items. To adapt the standard analysis to the diffuse adversary setting, we analyze the extent to which the adversary can assign probability to old items. Recall that old items that are not in the on-line algorithm's cache are called worrisome, as are requests to such items. We analyze the extent to which the adversary can cause worrisome requests.

We next sketch the argument for the upper bound, glossing over issues of probabilistic conditioning, in order to convey the intuition. In the subsequent section we give a formally correct treatment. We then give the lower bound; the intuition for the lower bound is similar to that of the upper bound.

Consider the $\ell$ th phase for any $\ell$. There are $k$ non-redundant requests in the phase (except possibly for the last phase, which may have fewer). Consider the state of any marking algorithm DMARK just before the $(i+1)$ st non-redundant request, for $1 \leq i \leq k-1$.

The $i$ redundant items are marked and in the cache. Of the $k$ items requested last phase, at most new_in_ph $(\ell)$ are worrisome (out of the cache). Thus, the adversary can assign at most $\epsilon$ new_in_ph $(\ell)$ probability to worrisome items. 
Since there are only $i$ redundant items, the adversary has to assign at least $1-\epsilon i$ probability to non-redundant items. Therefore, the probability that the request will be worrisome, given that the request turns out to be non-redundant, is at most

$$
\frac{\epsilon \text { new_in_ph }(\ell)}{1-\epsilon i}=\frac{\text { new_in_ph }(\ell)}{\epsilon^{-1}-i}
$$

(or 1 if this quantity is negative or more than 1). Summing over $i$, adding new_in_ph $(\ell)$ for the evictions due to new requests, and dividing by new_in_ph $(\ell) / 2$ (the amortized cost incurred by Opт for the phase) gives the desired upper bound $2 \Phi$ on the competitive ratio.

The above upper bound can be turned into a roughly equivalent lower bound. The lower bound loses a factor of 2 because of our use of new requests in approximating $\operatorname{Opт}(s)$. It loses an additional additive term of 1 in some cases; we revisit this issue after presenting the lower bound.

\section{$3 \quad$ Upper Bound for Deterministic Algorithms}

Next we prove the upper bounds on deterministic strategies in Theorem i:

Lemma 2 For any lazy deterministic marking algorithm DMARK and $D \in \Delta_{\epsilon}$,

$$
\mathrm{E}_{D}[\operatorname{DMARK}(r)] \leq 2 \Phi(\epsilon, k) \cdot \mathrm{E}_{D}[\operatorname{Opt}(r)]+O(1)
$$

Proof: Without loss of generality, assume that $D$ generates only sequences whose last phase has $k$ non-redundant requests. (Otherwise we can easily modify the distribution so that the condition is satisfied, while increasing $\mathrm{E}[\mathrm{OpT}(r)]$ by at most the constant $k$.) In the context of the random sequence $r$, define the following random variables and events.

$R_{\ell, i}$ - the $(i+1)$ st non-redundant request in the $\ell$ th phase of $r$, if there is an $\ell$ th phase.

prefix $(R)$ - the prefix of $r$ up to but not including request $R$ of $r$.

new_bef $(R)$ - the number of new requests before request $R$ in the phase of $r$ containing $R$.

new_in_ph $(\ell)$ - the total number of new requests made in the $\ell$ th phase of $r$, if there is an $\ell$ th phase, otherwise 0 .

worrisome $(R)$ - the event that request $R$ of $r$ is worrisome.

In what follows, we abuse notation slightly as follows. By the event "prefix $\left(R_{\ell, i}\right)=$ $s$ ", we mean "there is an $\ell$ th phase in $r$ and the prefix of $r$ preceding request $R_{\ell, i}$ is sequence $s$ ". Similarly, by the event "worrisome $\left(R_{\ell, i}\right)$ ", we mean "there is an $\ell$ th phase in $r$ and the request $R_{\ell, i}$ in that phase is worrisome".

We start by proving the following claim: 
Claim 1 Fix any $\ell$ and $i(1 \leq i \leq k-1)$. Let $s$ be any sequence such that the event $\operatorname{prefix}\left(R_{\ell, i}\right)=s$ can happen. That is, $s$ has $\ell$ phases, and the last phase of $s$ has $i$ non-redundant requests. Then

$\operatorname{Pr}\left[\operatorname{worrisome}\left(R_{\ell, i}\right) \mid \operatorname{prefix}\left(R_{\ell, i}\right)=s\right] \leq \mathrm{E}\left[\frac{\text { new_bef }\left(R_{\ell, i}\right)}{\max \left\{1, \epsilon^{-1}-i\right\}} \mid \operatorname{prefix}\left(R_{\ell, i}\right)=s\right]$.

Conditioning on "prefix $\left(R_{\ell, i}\right)=s$ " lets us use the restrictions on the adversary.

Here is the proof of Claim in the event that $s$ is a prefix of $r$, consider the random variable $r_{t}$ where $t=|s|+1$. (There must be such a request because $i<k$ and each phase of $r$, including the last, by the assumption at the beginning of the proof, has $k$ non-redundant requests.)

The event prefix $\left(R_{\ell, i}\right)=s$ happens if and only if $s$ is a prefix of $r$ and $r_{t}$ is non-redundant. If $\operatorname{prefix}\left(R_{\ell, i}\right)=s$, then the event worrisome $\left(R_{\ell, i}\right)$ happens if and only if $r_{t}$ is worrisome. Thus,

$$
\begin{aligned}
& \operatorname{Pr}\left(\text { worrisome }\left(R_{\ell, i}\right) \mid \operatorname{prefix}\left(R_{\ell, i}\right)=s\right) \\
& \quad=\operatorname{Pr}\left(\text { worrisome }\left(r_{t}\right) \mid s \text { is a prefix of } r \text { and } r_{t}\right. \text { is non-redundant) } \\
& \quad=\frac{\operatorname{Pr}\left(\text { worrisome }\left(r_{t}\right) \mid s \text { is a prefix of } r\right)}{\operatorname{Pr}\left(r_{t} \text { is non-redundant } \mid s \text { is a prefix of } r\right)} .
\end{aligned}
$$

Assume that $s$ is a prefix of $r$. After processing $s$, DMARK has all but new_bef $\left(r_{t}\right)$ of the items requested in the previous phase in the cache. Thus, the adversary can assign at most $\epsilon$ new_bef $\left(r_{t}\right)$ probability to worrisome items. Thus, the numerator above is at most $\epsilon$ new_bef $\left(r_{t}\right)$. Since there have been $i$ non-redundant requests in this phase before $r_{t}$, there are only $i$ redundant items, so the denominator above is at least $1-\epsilon i$. To finish the proof of Claim 11, note that E[new_bef $\left.\left(R_{\ell, i}\right) \mid \operatorname{prefix}\left(R_{\ell, i}\right)=s\right]=$ new_bef $\left(r_{t}\right)$.

Now fix $i$ and $\ell$. In the set of events $\left\{\operatorname{prefix}\left(R_{\ell, i}\right)=s \mid s\right.$ is a sequence\}, exactly one event happens. Thus, the bound in Claim 1 holds unconditionally:

$$
\operatorname{Pr}\left[\text { worrisome }\left(R_{\ell, i}\right)\right] \leq \mathrm{E}\left[\frac{\text { new_bef }\left(R_{\ell, i}\right)}{\max \left\{1, \epsilon^{-1}-i\right\}}\right] .
$$

Since new_bef $\left(R_{\ell, i}\right) \leq$ new_in_ph $(\ell)$, it follows that for all $\ell$ and $i$,

$$
\operatorname{Pr}\left[\operatorname{worrisome}\left(R_{\ell, i}\right)\right] \leq \mathrm{E}\left[\frac{\text { new_in_ph }(\ell)}{\max \left\{1, \epsilon^{-1}-i\right\}}\right] .
$$

Since $\operatorname{DMARK}(r)$ is the number of new or worrisome requests in $r$,

$$
\begin{aligned}
\operatorname{E}[\operatorname{DMaRK}(r)] & \leq \mathrm{E}\left[\sum_{\ell} \text { new_in_ph }(\ell)+\sum_{\ell, i} \frac{\text { new_in_ph } \left.(\ell)_{\max \left\{1, \epsilon^{-1}-i\right\}}\right]}{}\right. \\
& =\left(1+\sum_{i} \max \left\{1, \epsilon^{-1}-i\right\}^{-1}\right) \cdot \mathrm{E}\left[\sum_{\ell} \text { new_in_ph }(\ell)\right] \\
& =\Phi(\epsilon, k) \mathrm{E}[\operatorname{new}(r)] \\
& \leq \Phi(\epsilon, k) \mathrm{E}[\operatorname{Opt}(r) / 2] \quad \text { (by Lemma } 1) .
\end{aligned}
$$




\section{Lower Bound for Deterministic Algorithms}

Next we prove the lower bounds on deterministic strategies in Theorem 11:

Lemma 3 For any $\epsilon>0$, any $k$, and any deterministic on-line algorithm $A$, there is a distribution $D \in \Delta_{\epsilon}$ such that

$$
\mathrm{E}_{D}[A(r)] \geq(\Phi(\epsilon, k)-1+1 / m) \cdot \mathrm{E}_{D}[\mathrm{OPT}(r)] .
$$

where $m=\max \left\{1,\left\lceil\epsilon^{-1}\right\rceil-k\right\}$, and $\mathrm{E}_{D}[\mathrm{OPT}(r)]$ is arbitrarily large.

Proof: We describe $D$ by describing an adversary that requests items probabilistically subject to the limitations of $\Delta_{\epsilon}$. Fix $\epsilon>0$ and $k>0$. Assume $\epsilon>1 / 2 k$ (otherwise the desired lower bound is trivially satisfied, because $\Phi(1 / 2 k, k)-1+1 / m$ is less than 1$)$.

The adversary requests the items in an on-line fashion, phase by phase. In the first part of each phase, the adversary makes $m$ new requests by assigning probability only to items not previously requested.

For each remaining request, the adversary assigns a probability to each item as follows. First priority is given to worrisome items (those previously requested in this phase or in the last one but not in the cache of $A$ ). Second priority is given to redundant items (those requested previously in this phase and in the cache). Third priority is given to the remaining old items (the items not yet requested this phase, but in the cache).

Items are selected in order of priority and assigned as much probability as possible, subject to the constraint that no item is assigned probability more than $\epsilon$ and the total probability assigned is 1 . By the choice of $m$, we have $(k+m) \epsilon \geq 1$, so all three kinds of items suffice for all probability to be assigned.

The adversary follows this strategy until $k$ distinct items have been requested, at which point the adversary begins a new phase. The adversary continues for $N$ phases, where $N$ is arbitrarily large so that Орт $(r)$ is also arbitrarily large.

This defines the distribution $D \in \Delta_{\epsilon}$. Let $r$ be a random request sequence chosen from $D$. Next we prove that $\mathrm{E}[A(r)] \geq N m(\Phi(\epsilon, k)-1+1 / m)$. This proves the claimed bound, since Opt $(r) \leq N m$ (by Lemma 1 ). Consider any $\ell$ s.t. $1 \leq \ell \leq N$. For $i=m, \ldots, k-1$, define

worrisome $\left(R_{\ell, i}\right)$ - the event that the $i$ th non-redundant request of the $\ell$ th phase is worrisome.

The expectation of $A(r)$ is $N m+\sum_{\ell, i} \operatorname{Pr}\left[\operatorname{worrisome}\left(R_{\ell, i}\right)\right]$. For any $\ell$ and $i$ s.t. $m \leq i \leq k-1$, consider the time just before the $(i+1)$ st non-redundant request of the $\ell$ th phase. There have been $i$ non-redundant requests so far in the phase, so there are $i$ redundant items. There have been $m$ new requests 
so far, so there are $k+m$ items that were requested last phase or already this phase. Since the on-line algorithm has at least $m$ of these items not in the cache, there are at least $m$ worrisome items. Thus, the adversary assigns at least $\mathrm{\epsilon m}$ probability to worrisome items and at least $\epsilon i$ probability to redundant items. (Unless $\epsilon m+\epsilon i>1$, in which case $\operatorname{Pr}\left[\right.$ worrisome $\left.\left(R_{\ell, i}\right)\right]=1$ - the adversary forces a worrisome request.) Thus, the probability that the request is worrisome, conditioned on it being non-redundant, is

$\operatorname{Pr}\left[\operatorname{worrisome}\left(R_{\ell, i}\right)\right] \geq \frac{\epsilon m}{\max \{1-\epsilon i, \epsilon m\}}=\frac{m}{\max \left\{\epsilon^{-1}-i, m\right\}}=\frac{m}{\max \left\{\epsilon^{-1}-i, 1\right\}}$.

The rightmost equality holds because the choice of $m$ implies that either $m=1$ or $\epsilon^{-1}-i \geq m$. Adding the $m$ new requests and summing over $i=m, \ldots, k-1$, the expected cost to $A$ for each of the $N$ phases is at least

$$
m+\sum_{i=m}^{k-1} \frac{m}{\max \left\{\epsilon^{-1}-i, 1\right\}} \geq 1+\sum_{i=1}^{k-1} \frac{m}{\max \left\{\epsilon^{-1}-i, 1\right\}} .
$$

The rightmost expression is $m(\Phi(\epsilon, k)-1+1 / m)$.

The adversary can probably be made a little stronger to get a slightly better lower bound when $\epsilon \leq 1 /(k+1)$. In this case the issue of how the optimal adversary should fix $m$ appears to be relatively subtle. This is why the lower bound loses the additive 1 with respect to the upper bound in this case. One small improvement to the above adversary would be, when the adversary is requesting new items, to use the opportunity to also allocate probability to worrisome items.

\section{$5 \quad$ Randomized Strategies}

In this section we finish the proof of Theorem 1 by proving the upper and lower bounds for randomized strategies claimed there. By using what we already know, very little work is required to get the bounds.

We first consider lower bounds. Fix $\epsilon>0$ and $k>0$. We start with the case $\epsilon \leq 1 /(k+1)$. For simplicity we make the technical assumption that $\epsilon^{-1}$ is an integer. This assumption is not too restrictive and allows us to reuse the deterministic lower bound as follows.

Lemma 4 If $\epsilon^{-1}$ is an integer greater than $k$, then the distribution $D$ described in the proof of Lemma 司is independent of the algorithm $A$.

Proof: Consider that distribution. Within each phase, the random sequence $r$ has requests to $m$ new items, followed by requests restricted to a set of $k+m$ items, where $m=\max \left\{1, \epsilon^{-1}-k\right\}$, until $k$ distinct items have been requested. The condition on $\epsilon$ and the choice of $m$ imply that $m=\epsilon^{-1}-k$, so that $\epsilon=1 /(k+m)$. In this case, each phase simply consists of requests to $m$ new 
items, followed by a sequence of requests to the $k+m$ items, where each request is chosen uniformly at random from those $k+m$ items, until a total of $k$ distinct items have been requested, after which the next phase begins.

This distribution generalizes a distribution defined in a previous lower bound on the competitive ratio of randomized on-line strategies against the standard adversary [2, Thm. 8.7], 13, Thm. 13.2]. (That lower bound is equivalent to our case $m=1$.) There and here, Yao's principle implies that for a random input $r$ from any input distribution $D$, any randomized on-line algorithm $A_{R}$ satisfies

$$
\mathrm{E}\left[A_{R}(r)\right] \geq \inf _{A} \mathrm{E}[A(r)]
$$

where $A$ ranges over all deterministic on-line algorithms.

(Briefly, this is because $A_{R}$ may be viewed as probabilistically picking some deterministic algorithm $A$, and then running $A$ on the input $r$. Thus, $\mathrm{E}_{D}\left[A_{R}(r)\right]=$ $\sum_{A} \operatorname{Pr}\left[A_{R}\right.$ chooses $\left.A\right] \cdot \mathrm{E}_{D}[A(r)] \geq \inf _{A} \mathrm{E}_{D}[A(r)]$. Here $D$ can be any distribution, but we take it to be the one defined in Lemma 3. The input $r$ is randomly chosen from $D$. We refer the reader to [13, Thm. 13.2] or [2, Thm. 8.7] for a full explanation of Yao's principle in this context.)

By Lemma 4, in the special case when $\epsilon^{-1}$ is an integer greater than $k$, the distribution defined in the previous section is independent of the on-line algorithm $A$. Thus, by Yao's principle, the lower bounds proved there extend to randomized algorithms. This proves:

Lemma 5 Suppose $\epsilon \leq 1 /(k+1)$ and $\epsilon^{-1}$ is an integer. Then the lower bound established in Lemma 3 also applies to randomized on-line algorithms.

Decreasing $\epsilon$ only weakens the adversary. Thus, when $\epsilon$ is not an integer, letting $\epsilon^{\prime}=1 /\left\lceil\epsilon^{-1}\right\rceil<\epsilon$, the lower bounds hold with $\epsilon^{\prime}$ replacing $\epsilon$.

Also, when $\epsilon=1 /(k+1)$ it is easy to verify that the above lemma implies that the ratios are at least $H(k) \doteq \sum_{1}^{k} 1 / i$. This proves:

Lemma 6 Suppose $\epsilon \geq 1 /(k+1)$. Then $\mathcal{R} \mathcal{R}\left(\Delta_{\epsilon}\right) \geq H(k)$.

So the above two lemmas prove the lower bounds for randomized strategies claimed in Theorem 1. What about the upper bounds? Because the diffuse adversary is no stronger than the standard adversary, we get immediately from previous results that:

Lemma 7 For $\epsilon \leq 1 /(k+1), \mathcal{R} \mathcal{R}\left(\Delta_{\epsilon}\right.$, RMARK $) \leq 2 \Phi(\epsilon, k)$.

For $\epsilon \geq 1 /(k+1), \mathcal{R} \mathcal{R}\left(\Delta_{\epsilon}\right.$, RMARK $) \leq 2 H(k)-1$, while $\mathcal{R} \mathcal{R}\left(\Delta_{\epsilon}\right.$, PARTITION $) \leq$ $H(k)$, and $\mathcal{R} \mathcal{R}\left(\Delta_{\epsilon}\right.$, EQUitable $) \leq H(k)$.

The first upper bound follows from the fact that Lemma 2 also applies to RMARK (since the upper bound applies to any deterministic marking algorithm, i.e., any conditioning of RMARK on a particular outcome of its random choices). The remaining upper bounds follow from known upper bounds on the competitive ratios of the various algorithms against the (stronger) standard adversary [2, 月, 12, 1]. Lemma 7 proves the upper bounds on randomized strategies in Theorem 1. This completes the proof of that theorem. 


\section{Alternate Proof that LRU is Optimal}

For the record, we include here a "distillation" of Koutsoupias and Papadimitriou's proof that LRU is optimal against the diffuse adversary $\Delta_{\epsilon}$. This version of the proof is shorter and self-contained, but does not give the intermediate results about work functions in the original proof.

Given a request sequence $s$ of items from a universe $U$, and an (arbitrary) initial ordering $\pi$ of the items, define the rank of an item $x \in U$ in $s$ to be the rank of $x$ in the following ordering: items that are requested in $s$ are first, in order of last request; items that are not requested in $s$ are next, ordered by $\pi$.

In analyzing an on-line paging algorithm, if $s$ is the sequence of requests seen so far, then the most recently requested item currently has rank 1 , the next most recently requested item currently has rank 2, etc. Without loss of generality, when specifying a request or the contents of the cache, we can specify each item by its current rank; this uniquely identifies the item. Except in the proof of Lemma 8 where we use both representations, items in this section are assumed to be specified by their current rank.

Lemma 8 Let $r$ and $r^{\prime}$ be two equal-length request sequences. Let $r$ and $\mathbf{r}^{\prime}$, respectively, be the same sequences but with each request specified by rank (w.r.t. the same initial ordering and universe). If $r$ dominates $r^{\prime}$ in the sense that $r_{t} \geq r_{t}^{\prime}$ for all $t$, then $\operatorname{OpT}(r) \geq \operatorname{OpT}\left(r^{\prime}\right)$.

Proof: It suffices to prove the case when there is a single $d$ such that $r_{d}^{\prime}=r_{d}-1$ but $r_{t}=\mathbf{r}_{t}^{\prime}$ for all $t \neq d$. The general case then follows by induction. Assume such a $d$.

How do $r$ and $r^{\prime}$ differ? Consider the two sequences simultaneously for $t=1,2, \ldots,|r|$ in an on-line fashion. At each $t$ focus on the ranks of the items in the two subsequences $s=r_{1} r_{2} \ldots r_{t}$ and $s^{\prime}=r_{1}^{\prime} r_{2}^{\prime} r^{\prime} \ldots r_{t}^{\prime}$.

At each time $t<d$, for each item, the rank in $s$ equals the rank in $s^{\prime}$. Let $x$ and $x^{\prime}$ be the items requested, respectively, in $r$ and $r^{\prime}$ at time $d$. By assumption, just before time $d$, the respective ranks of $x$ and $x^{\prime}$ are $r_{d}$ and $r_{d}-1$. What about just after time $d$ ? In sequence $s$, the rank of $x$ changes to 1 , while the rank of $x^{\prime}$ changes to $\mathrm{r}_{d}$. In sequence $s^{\prime}$, the rank of $x$ stays $\mathrm{r}_{d}$, while the rank of $x^{\prime}$ changes to 1 . For each item other than $x$ or $x^{\prime}$, the rank of the item is equal in both sequences.

This means that the sequence of items requested by $r$ is the same as the sequence of items requested by $r^{\prime}$, except that from time $d$ to the end, the roles of $x$ and $x^{\prime}$ are reversed: if $r$ requests $x$ (resp. $x^{\prime}$ ), then $r^{\prime}$ requests $x^{\prime}$ (resp. $x$ ).

Let $i$ and $i^{\prime}$, respectively, be the times of the most recent requests to $x$ and $x^{\prime}$ before time $d$. (If either item is being requested for the first time, then let $i$ or $i^{\prime}$ equal 1, as appropriate.) By assumption $\mathrm{r}_{d}^{\prime}=\mathrm{r}_{d}-1$, so $i \leq i^{\prime}$.

Consider any schedule $S$ for $r$. For any $j$ with $i^{\prime}<j \leq d$, consider obtaining $S^{\prime}$ from $S$ by reversing the roles of $x$ and $x^{\prime}$ from time $j$ onward (i.e. swapping the two in $\left.S_{j}, S_{j+1}, \ldots\right)$. By the established relation between $r$ and $r^{\prime}, S^{\prime}$ will be a valid schedule for $r^{\prime}$. To finish, we need only choose $j$ so that $S^{\prime}$ costs no more 
than $S$. In particular, at time $j, S^{\prime}$ should evict no more of the two pages $\left\{x, x^{\prime}\right\}$ than $S$ does. If for some $j,\left|\left\{x^{\prime}, x\right\} \cap S_{j}\right| \in\{0,2\}$ or $\left|\left\{x^{\prime}, x\right\} \cap S_{j-1}\right| \in\{0,2\}$, then this $j$ clearly suffices. Otherwise there is a $j$ such that $\left\{x^{\prime}, x\right\} \cap S_{j-1}=\left\{x^{\prime}\right\}$ and $\left\{x^{\prime}, x\right\} \cap S_{j}=\{x\}$. Using this $j, S^{\prime}$ is cheaper than $S$.

Theorem 2 ([10]) Let $D$ be any distribution $D \in \Delta_{\epsilon}$. Let $A$ be any deterministic on-line algorithm. Then there is a distribution $D^{\prime} \in \Delta_{\epsilon}$ such that

$$
\mathrm{E}_{D}[\operatorname{Lru}(r)] \leq \mathrm{E}_{D^{\prime}}\left[A\left(r^{\prime}\right)\right] \text { and } \mathrm{E}_{D}[\mathrm{OPT}(r)] \geq \mathrm{E}_{D^{\prime}}\left[\mathrm{OPT}\left(r^{\prime}\right)\right] \text {, }
$$

where $r$ and $r^{\prime}$ are randomly chosen according to $D$ and $D^{\prime}$, respectively.

Thus, $\mathcal{R}\left(\Delta_{\epsilon}\right)=\mathcal{R}\left(\Delta_{\epsilon}\right.$, LRU $)$.

Proof: In what follows, we assume all items are specified not by name but by rank (with respect to some sequence implicit in context, the universe $U$ of the items requested by $D$, and an arbitrary initial ordering).

Intuitively, the argument is the following. At each request, we pair each page $\mathrm{x}$ in $A$ 's cache but not in LRU's cache with a unique page $f(\mathrm{x})$ in LRU's cache but not in $A$ 's. For each such $\mathrm{x}$, if $D$ assigns more probability to $\mathrm{x}$ than to $f(\mathrm{x})$, then we shift some of the probability from $\mathrm{x}$ to $f(\mathrm{x})$. This gives us a modified assignment of probabilities to pages for the request; in this way we define $D^{\prime}$. We show that this shifting procedure ensures that at each request, $A$ is as likely to fault (on a request from $D^{\prime}$ ) as LRU was (on the corresponding request from $D$ ). Furthermore, $D^{\prime}$ is better for Opt than $D$ is, because when we shift probability from $\mathrm{x}$ to $f(\mathrm{x})$, we know that, as $\mathrm{x}$ is not in LRU's cache but $f(\mathrm{x})$ is, we are shifting probability from a higher-ranked page to a lower-ranked page (in the sense of Lemma \&).

Formally, the following random experiment defines the distribution $D^{\prime}$ by describing how to choose a random sequence $r^{\prime}$ according to that distribution. Choose a random sequence $r$ according to $D$. Reveal $r$ in an on-line fashion, one request at a time, producing each corresponding request of $r^{\prime}$ as follows.

Let $L$ denote the cache of LRU (specified by rank with respect to $s$ ) after processing $\mathrm{s}=\mathrm{r}_{1} \ldots \mathrm{r}_{t-1}$. Similarly, let $\mathrm{A}$ denote the cache (specified by rank with respect to $\mathrm{s}^{\prime}$ ) of $A$ after processing $\mathrm{s}^{\prime}=\mathrm{r}_{1}^{\prime} \ldots \mathrm{r}_{t-1}^{\prime}$. Let $f$ be any 1-1 mapping from $A-L$ into $L-A$ (note $|A| \leq|L|$ ) and define (in the context of $s$ and $s^{\prime}$ )

$$
\begin{aligned}
\mathcal{X} & \doteq\{\mathrm{x} \in \mathrm{A}-\mathrm{L} \mid p(f(\mathrm{x}))<p(\mathrm{x})\}, \text { where } \\
p(\mathrm{x}) & \doteq \operatorname{Pr}_{D}(\mathrm{x} \mid \mathrm{s}) .
\end{aligned}
$$

$\mathcal{X}$ is the set of pages from which we want to shift probability.

Finally, determine $r_{t}$ as follows. First set $r_{t}^{\prime}=r_{t}$, but if $r_{t} \in \mathcal{X}$, change $r_{t}^{\prime}$ to $f\left(\mathrm{r}_{t}\right)$ with probability $p\left(f\left(\mathrm{r}_{t}\right)\right) / p\left(\mathrm{r}_{t}\right)$.

This completes the random experiment that gives $r^{\prime}$ and so defines $D^{\prime}$. Each outcome of this experiment determines a pair of random variables $\left(r, r^{\prime}\right)$.

We use " $\operatorname{Pr}_{D^{\prime}}\left(X \mid \mathbf{s}, \mathbf{s}^{\prime}\right)$ " to denote the probability of event $X$ conditioned on $s$ and $s^{\prime}$ being prefixes of $r$ and $r^{\prime}$. The following claim characterizes the distribution of $r_{t}^{\prime}$ conditioned on this event. 
Claim 2 Fix any two sequences $\mathrm{s}$ and $\mathrm{s}^{\prime}$ with length $t-1$. Define $p^{\prime}(\mathrm{x}) \doteq$ $\operatorname{Pr}_{D^{\prime}}\left(\mathrm{r}_{t}^{\prime}=\mathrm{x} \mid \mathrm{s}, \mathrm{s}^{\prime}\right)$. Then for each $\mathrm{x}, p^{\prime}(\mathrm{x})=p(\pi(\mathrm{x}))$, where $\pi$ is the permutation defined by $\pi(\mathrm{x})=\mathrm{x}$ unless $\mathrm{x} \in \mathcal{X}$ or $f(\mathrm{x}) \in \mathcal{X}$, in which case $\pi(\mathrm{x})=f(\mathrm{x})$ and $\pi(f(\mathrm{x}))=\mathrm{x}$.

The claim follows by direct calculation based on the last line of the experiment.

Claim 3 Let $\mathrm{r}, \mathrm{r}^{\prime}, \mathrm{s}$, and $\mathrm{s}^{\prime}$, be as in Claim 娄. Then

$$
\underset{D^{\prime}}{\operatorname{Pr}}\left[\operatorname{LRU} \text { faults on } \mathrm{r}_{t} \mid \mathrm{s}, \mathrm{s}^{\prime}\right] \leq \operatorname{Pr}_{D^{\prime}}\left[\text { A faults on } \mathrm{r}_{t}^{\prime} \mid \mathrm{s}, \mathrm{s}^{\prime}\right] \text {. }
$$

Why? It suffices to show that for every item $\mathrm{x}$ in $\mathrm{A}$, there is a unique item $\mathrm{y}$ in $\mathrm{L}$ such that $p^{\prime}(\mathrm{x}) \leq p(\mathrm{y})$. But by Claim 2 and the choice of $\mathcal{X}$, this is the case: take $\mathrm{y}=\mathrm{x}$ unless $\mathrm{x} \in \mathrm{A}-\mathrm{L}$, in which case take $\mathrm{y}=f(\mathrm{x}) \in \mathrm{L}-\mathrm{A}$.

Note that in Lemma 3, equality does not necessarily hold because $A$ may not have $k$ pages in its cache, or it may have "irrelevant" pages in its cache - a page $\mathrm{x}$ that $D$ requests with less probability than the corresponding page $f(\mathrm{x})$ in LRU's cache (so no probability is shifted from $\mathrm{x}$ to $f(\mathrm{x})$ ).

Claim 4 The first part of the theorem is true: $\mathrm{E}_{D}[\mathrm{LRU}(\mathrm{r})] \leq \mathrm{E}_{D^{\prime}}\left[A\left(\mathrm{r}^{\prime}\right)\right]$.

This follows directly from Claim 3. To see it formally, letting $s$ and $s^{\prime}$ range over all equal-length pairs of sequences, we have

$$
\begin{aligned}
\mathrm{E}_{D}[\operatorname{LrU}(\mathbf{r})] & =\sum_{\mathbf{s}, \mathbf{s}^{\prime}} \operatorname{Pr}_{D^{\prime}}\left(\mathbf{s}, \mathbf{s}^{\prime}\right) \underset{D^{\prime}}{\operatorname{Pr}}\left[\operatorname{LRU} \text { faults on } \mathbf{r}_{|\mathbf{s}|+1} \mid \mathbf{s}, \mathbf{s}^{\prime}\right] \\
& \leq \sum_{\mathbf{s}, \mathbf{s}^{\prime}} \operatorname{Pr}_{D^{\prime}}\left(\mathbf{s}, \mathbf{s}^{\prime}\right) \underset{D^{\prime}}{\operatorname{Pr}}\left[A \text { faults on } \mathbf{r}_{\left|\mathbf{s}^{\prime}\right|+1}^{\prime} \mid \mathbf{s}, \mathbf{s}^{\prime}\right] \\
& =\mathrm{E}_{D^{\prime}}\left[A\left(\mathbf{r}^{\prime}\right)\right] .
\end{aligned}
$$

Above $\operatorname{Pr}_{D^{\prime}}\left(\mathbf{s}, \mathbf{s}^{\prime}\right)$ denotes the probability that $\mathrm{s}$ is a prefix of $r$ and $\mathbf{s}^{\prime}$ is a prefix of $r^{\prime}$ in the random experiment.

Claim 5 The second part of the theorem is true: $\mathrm{E}_{D}[\mathrm{OPT}(\mathrm{r})] \geq \mathrm{E}_{D^{\prime}}\left[\mathrm{OPT}\left(\mathrm{r}^{\prime}\right)\right]$.

Since the random experiment described above produces the same distribution on $r$ as $D$ does, it suffices to prove the inequality assuming that the pair $\left(r, r^{\prime}\right)$ is generated by that experiment. Since LRU keeps the most recently requested items in its cache, and $f:(\mathrm{A}-\mathrm{L}) \rightarrow(\mathrm{L}-\mathrm{A})$, we have $\mathrm{x} \leq f(\mathrm{x})$. Thus, in any outcome, $r$ dominates $r^{\prime}$ (in the sense of Lemma 8) and so Opt $(r) \geq \operatorname{Opt}\left(r^{\prime}\right)$. This proves the claim.

Claim 6 The distribution $D^{\prime}$ defined by the random experiment is in $\Delta_{\epsilon}$.

This also follows directly from Claim 2. To prove it in detail, we need to show that for any $s^{\prime}$ and $x, \operatorname{Pr}_{D^{\prime}}\left(x \mid s^{\prime}\right) \leq \epsilon$. But

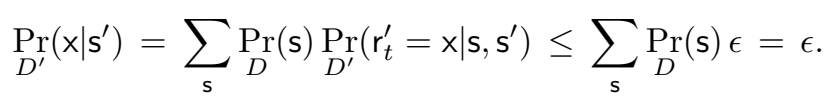


Above $\operatorname{Pr}_{D}(\mathrm{~s})$ denotes the probability that $\mathrm{s}$ is a prefix of $\mathrm{r}$, and $\mathrm{s}$ ranges over all sequences of length $\left|s^{\prime}\right|=t-1$. The second-to-last inequality follows because by Claim 2 each $\operatorname{Pr}_{D^{\prime}}\left(\mathrm{r}_{t}^{\prime}=\mathrm{x} \mid \mathrm{s}, \mathrm{s}^{\prime}\right)$ equals $\operatorname{Pr}_{D}(\mathrm{y} \mid \mathrm{s})$ for some $\mathrm{y}$, and by the assumption that $D \in \Delta_{\epsilon}, \operatorname{Pr}_{D}(\mathrm{y} \mid \mathrm{s}) \leq \epsilon$. This proves the claim (and the theorem!).

\section{Acknowledgements}

Thanks to Elias Koutsoupias and Lenny Ng for helpful discussions and suggestions. Thanks to the anonymous referees for helping to clarify the presentation.

\section{References}

[1] Dimitris Achlioptas, Marek Chrobak, and John Noga. Competitive analysis of randomized paging algorithms. In Josep Díaz and Maria Serna, editors, Algorithms-ESA '96, Fourth Annual European Symposium, volume 1136 of Lecture Notes in Computer Science, pages 419-430, Barcelona, Spain, 25-27 September 1996. Springer.

[2] Allan Borodin and Ran El-Yaniv. Online Computation and Competitive Analysis. Cambridge University Press, 1998.

[3] Allan Borodin, Sandy Irani, Prabhakar Raghavan, and Baruch Schieber. Competitive paging with locality of reference. Journal of Computer and System Sciences, 50(2):244-258, April 1995.

[4] Amos Fiat and Anna R. Karlin. Randomized and multipointer paging with locality of reference. In Proceedings of the Twenty-Seventh Annual ACM Symposium on Theory of Computing, pages 626-634, Las Vegas, Nevada, 29 May-1 June 1995.

[5] Amos Fiat, Richard M. Karp, Michael Luby, Lyle A. McGeoch, Daniel D. Sleator, and Neal E. Young. Competitive paging algorithms. Journal of Algorithms, 12(4):685-699, December 1991.

[6] Amos Fiat and Ziv Rosen. Experimental studies of access graph based heuristics: Beating the LRU standard? In Proceedings of the Eighth Annual ACM-SIAM Symposium on Discrete Algorithms, pages 63-72, New Orleans, Louisiana, 5-7 January 1997.

[7] P. A. Franaszek and T. J. Wagner. Some distribution-free aspects of paging algorithm performance. J. Assoc. Comput. Math., 21:31-39, 1974.

[8] Sandy Irani, Anna R. Karlin, and Steven Phillips. Strongly competitive algorithms for paging with locality of reference. SIAM Journal on Computing, 25(3):477-497, June 1996.

[9] Anna R. Karlin, Steven J. Phillips, and Prabhakar Raghavan. Markov paging (extended abstract). In 33rd Annual Symposium on Foundations of Computer Science, pages 208-217, Pittsburgh, Pennsylvania, 24-27 October 1992. IEEE.

[10] Elias Koutsoupias and Christos H. Papadimitriou. Beyond competitive analysis. In Proc. of the 35th IEEE Annual Symp. on Foundation of Computer Science, pages 394-400, 1994. 
[11] Carsten Lund, Steven Phillips, and Nick Reingold. IP over connection-oriented networks and distributional paging. In 35th Annual Symposium on Foundations of Computer Science, pages 424-434, Santa Fe, New Mexico, 20-22 November 1994. IEEE.

[12] Lyle A. McGeoch and Daniel D. Sleator. A strongly competitive randomized paging algorithm. Algorithmica, 6(6):816-825, 1991.

[13] Rajeev Motwani and Prabhakar Raghavan. Randomized Algorithms. Cambridge University Press, 1995.

[14] Daniel D. Sleator and Robert E. Tarjan. Amortized efficiency of list update and paging rules. Comm. ACM, 28(2):202-208, February 1985.

[15] Neal E. Young. Competitive paging and dual-guided algorithms for weighted caching and matching. (Thesis) Tech. Rep. CS-TR-348-91, Computer Science Department, Princeton University, October 1991.

[16] Neal E. Young. The $k$-server dual and loose competitiveness for paging. Algorithmica, 11(6):525-541, 1994.

[17] Neal E. Young. On-line file caching. In Proceedings of the Ninth Annual ACMSIAM Symposium on Discrete Algorithms, pages 82-86, San Francisco, California, 25-27 January 1998. 